\title{
QUEDA EM ADULTOS IDOSOS: CONSIDERAÇÕES SOBRE A REGULAÇÃO DO EQUILÍBRIO, ESTRATÉGIAS POSTURAIS E EXERCÍCIO FÍSICO
}

\author{
Fall in older adults: considerations on balance regulation, postural \\ strategies, and physical exercise
}

Marcelo de Maio Nascimento ${ }^{\text {(i) }}$

Quedas são comuns entre a população idosa, consideradas como questão de saúde pública, responsáveis por alto grau de morbidade e mortalidade. Isso evidencia a realização de estudos teóricos que qualifiquem o entendimento sobre a relação entre queda e seus fatores de risco; a fisiopatologia dos distúrbios do equilíbrio e da marcha; e a identificação precoce da "queda idiopática" (sem causa evidente). Assim, com base na literatura especializada, o presente estudo apresenta uma visão geral sobre o papel dos sistemas sensoriais responsáveis pela regulação do controle postural, evidenciando as principais estratégias utilizadas por idosos para a manutenção do controle postural e os benefícios proporcionados pela prática regular do exercício físico para a prevenção de quedas de idosos. Como resultado, é apresentado um modelo ilustrativo sobre a sequência de estratégias utilizadas por idosos para reestabelecer a posição ereta em diferentes fases de perturbação postural. Conclui-se que o baixo desempenho da atenção, da memória e de funções executivas eleva a instabilidade postural e reduz a velocidade da marcha, aumentando o risco de quedas. Nesse contexto, a prática regular de exercícios físicos é capaz de reduzir significativamente a taxa de queda de idosos. Aconselham-se a prática de exercícios programados, que agreguem tarefas cognitivas com grau de desafio da instabilidade de moderado a alto, e a prática semanal de duas a três horas.

PALAVRAS-CHAVE: acidentes por quedas; equilíbrio postural; cognição; envelhecimento.

Common among the older population, falls are considered a public health issue, accounting for high rates of morbidity and mortality. Thus, there is a need for theoretical studies that improve the understanding of the relationship between falls and their risk factors; the pathophysiology of balance and gait disorders; and early identification of "idiopathic fall" (with no apparent

$\vdash \quad$ cause). Based on the specialized literature, the present study provides an overview of the role of sensory systems responsible for regulating postural control, showing the main strategies that older adults use to maintain postural control and the benefits of regular physical exercise for prevention of falls in older adults. Then, a graphic model describing the sequence of strategies used by older adults to regain an upright stance in different phases of postural perturbation is presented. In conclusion, poor performance of attention, memory, and executive functions increases postural instability and reduces gait speed, thereby raising the risk of falls. In this context, regular physical exercise may significantly reduce the rate of falls in older adults. Planned exercises are recommended and should involve cognitive tasks with moderate-to-high level of instability for two to three hours/week.

KEYWORDS: accidental falls; postural balance; cognition; aging. 


\section{INTRODUÇÃO}

Nas últimas décadas, grande número de investigações foram desenvolvidas sobre o tema queda de idosos. A busca no PubMed pelos termos "fall elderly" ou "falls risk", no período de 2008 a 2018, resultou em 15.442 e 12.050 estudos, respectivamente. Não obstante, observa-se para o mesmo período 8.616 publicações relacionadas à prevenção de quedas (falls prevention). Tudo isso mostra a preocupação de diferentes áreas da saúde com a questão queda de adultos idosos.

Conforme a literatura especializada, estima-se que aproximadamente $30 \%$ da população idosa sofra uma queda a cada ano, ${ }^{1,2}$ existindo maior risco para o sexo feminino. ${ }^{3}$ Diante disso, a Organização Mundial da Saúde ${ }^{4}$ considera o caso como uma questão de saúde pública, pois quedas são responsáveis por lesões e fraturas. Por conseguinte, quedas afetam significativamente a autonomia do indivíduo, baixando a qualidade de vida percebida. ${ }^{5}$ Outra questão associada à queda de idosos incide no contingente hospitalizações e custos decorrentes. Em estudo realizado por Barros et al. ${ }^{6}$ junto ao banco de dados do Ministério da Saúde brasileiro foram identificadas 399.681 internações de idosos para o período de 2005 a 2010, o que gerou custo de $\mathrm{R} \$ 464.874 .275,91$ para serviços de autorização de internação hospitalar.

A literatura especializada atribui a causa de quedas a um conjunto de fatores classificados em extrínsecos e intrínsecos. ${ }^{7,8}$ Os primeiros estão relacionados às condições de infraestrutura do meio ambiente, incidindo em aspectos circunstanciais do piso e iluminação dos locais, o que se torna um perigo à segurança dos passantes. Enquanto fatores intrínsecos estão associados às transformações fisiológicas que atingem o organismo humano, decorrentes do avanço da idade. ${ }^{9}$ Assim, considerando os fatos, é importante a identificação precoce dos riscos de queda de idosos. Por outro lado, essa tarefa é complexa, uma vez que a queda é uma questão multifatorial. ${ }^{2}$

Por essa razão, aconselha-se a associação entre diferentes instrumentos e/ou escalas. ${ }^{78} \mathrm{O}$ mesmo ocorre com os procedimentos de prevenção de quedas, faz-se necessário o uso de distintas metodologias. Entre elas, sugere-se a conscientização do idoso sobre os perigos (fatores extrínsecos), o que pode ser atingido por meio de atividades educativas, favorecendo a transformação de hábitos de vida, o que pode tornar o indivíduo mais cauteloso. Uma segunda estratégia consiste em motivar o idoso à prática de exercícios físicos, o que amplia seus níveis de aptidão física. ${ }^{8}$

Com o envelhecimento, o organismo sofre uma série de alterações neurodegenerativas que irão prejudicar significativamente a eficiência do controle neuromuscular e os sistemas responsáveis pelos ajustes posturais em situações de desequilíbrio. ${ }^{10}$ Por essa razão, comparativamente, indivíduos idosos são mais suscetíveis à queda do que adultos jovens saudáveis. ${ }^{11}$ Entre as principais transformações responsáveis pelo aumento do risco de queda, consideram-se: as alterações do funcionamento do sistema sensorial e motor; ${ }^{12}$ o déficit do equilíbrio estático e dinâmico; ${ }^{8}$ a perda da força e da potência muscular dos membros inferiores; ${ }^{3}$ alterações no padrão da marcha; ${ }^{10}$ baixa dos níveis de flexibilidade; ${ }^{10,12}$ déficit da condição cognitiva $;{ }^{13}$ perda da acuidade visual $;{ }^{14,15}$ além do próprio medo de cair. ${ }^{16}$

Nesse contexto, sabe-se que a prática regular de exercícios físicos apropriados é capaz de manter ou mesmo desenvolver a condição física e cognitiva de indivíduos idosos, protegendo-os de cair. ${ }^{17,18}$ Estudos de revisão sistemática e metanálise mostraram que o exercício físico é um mecanismo eficaz e de baixo custo, útil à prevenção de quedas. ${ }^{16,17}$ Segundo os autores, os achados podem ser generalizados para grande parte da população idosa. Ademais, o exercício físico é bem aceito por essa população, trazendo também como benefício o aumento do contato social, o que fortalece vínculos.

Entretanto, de acordo com Fasano et al., ${ }^{19}$ em estudo intitulado "The neurobiology of falls", a abordagem da queda não pode ser orientada unicamente à doença. Para os autores a situação carece, igualmente, do entendimento anterior do processo fisiopatológico responsável pela queda. Porque mesmo que a chance de queda de um indivíduo saudável, acima de 65 anos, sem histórico de quedas, seja de $27 \%$, sabe-se que o único fator preditor para quedas futuras é o próprio histórico de queda anterior, porém essa evidência é insuficiente para a admissão de qualquer prevenção primária. ${ }^{19} \mathrm{O}$ fato destaca a importância do desenvolvimento de estudos que permitam a identificação dos indivíduos classificados como "caidores idiopáticos", ou seja, aqueles que caem mesmo na ausência de algum pretexto evidente ou enfermidade subjacente. ${ }^{19}$

O presente estudo teve por objetivo, com base na literatura especializada, apresentar aspectos teóricos sobre o papel dos sistemas sensoriais responsáveis pela regulação do controle postural, destacar as diferentes estratégias utilizadas por idosos para a manutenção do equilíbrio, além de exibir o potencial da prática regular do exercício físico na prevenção de quedas da população idosa.

\section{MÉTODO}

Trata-se de uma revisão integrativa da literatura especializada referente ao tema queda de idosos, com especial foco em três tópicos: sistemas sensoriais responsáveis pela regulação do equilíbrio corporal; estratégias adotadas pela população adulta idosa para a prevenção de quedas; e os benefícios do exercício físico estruturado na redução do risco 
de queda. As buscas foram realizadas nas bases de dados SciELO, MEDLINE, LILACS e Google Acadêmico com base nos seguintes descritores: queda idosos (fall elderly), risco de queda (fall risk) e prevenção de queda (falls prevention); estabelecendo como critérios:

- publicações em português e inglês;

- idade da população $\geq 60$ anos.

\section{QUEDA E CONTROLE POSTURAL}

O evento da queda é definido como a alteração da posição do indivíduo para um nível inferior à sua posição atual, seguida pelo contato não intencional com a superfície de apoio, sem que exista a imposição de um fator intrínseco ou acidente inevitável. ${ }^{20}$ Entre os fatores intrínsecos responsáveis por quedas, um dos mais complexos, contudo possível de ser tratado por meio da prática de exercícios físicos, é o controle postural (equilíbrio). ${ }^{5,12-16}$ Déficits nessa capacidade motora aumentam significativamente o risco de queda, ${ }^{1,2}$ principalmente junto à população idosa.,

A regulação do controle postural é considerada um sistema de entrada tripla com saída única de informações. ${ }^{12}$ Inicialmente, por meio dos sistemas somatossensorial, visual e vestibular, há a captação dos dados posturais referentes aos posicionamentos do corpo e suas extremidades no espaço; e o processamento desses ocorre no sistema nervoso central (SNC). ${ }^{15}$ Sendo assim, o SNC é o responsável pela integração dos subsídios posturais, que são transformados em importantes informações. Com base nessas informações o SNC emite uma série de ajustes posturais às extremidades do corpo: a ação é essencial à manutenção do centro de gravidade sobre a base de suporte do corpo. ${ }^{21}$

Vale salientar que os procedimentos aplicados à integração dos dados posturais são efetivados no cérebro, cerebelo, núcleos da base e tronco cerebral. ${ }^{9}$ Esse último é composto de vias ascendentes e descendentes, de onde partem comandos à regulação do tônus (comando postural), que é executado por meio de reflexos motores. Caso o funcionamento dos sistemas de regulação do equilíbrio (propriocepção, visão, audição) apresentem déficits parciais ou totais, o SNC fica impedido de traçar, a tempo, estratégias corretivas. Isso significa dizer que limitações nos sistemas de regulação sensorial podem inviabilizar os ajustes posturais, aumentando consideravelmente o risco de queda. ${ }^{9-11}$

Estudos de revisão sistemática ${ }^{22}$ e metanálise ${ }^{7}$ descreveram que o controle do equilíbrio estático e dinâmico é um processo complexo, visto que a ação, além de exigir a eficácia dos três sistemas sensoriais, também exige o funcionamento adequado do sistema motor e da velocidade do processamento cognitivo (funções executivas). ${ }^{13,19}$ Paralelo ao fato, deve-se considerar que a manutenção da postura ereta também é influenciada pela morfologia corporal, o que inclui variáveis como estatura, comprimento dos pés e distribuição do peso corporal. ${ }^{9}$

\section{REGULACCÃO DO EQUILÍBRIO E ESTRATEGIAS POSTURAIS}

Em geral, quatro estratégias são aplicadas à manutenção do equilíbrio corporal: tornozelo, quadril, passo e braços. A Figura 1 ilustra, de forma sequencial, quatro táticas executadas pelo indivíduo para evitar o desequilíbrio e a queda.

As situações destacadas na Figura 1 incidem em medidas adaptativas executadas pelo SNC para selecionar a estabilização postural (estratégia) mais apropriada em três situações: normal, perturbação, próximo à queda e queda. Os recursos empregados para o ajuste da postura podem ser descritos da seguinte forma:

- Fase 1a (estratégia do tornozelo): ao detectar, em situação tardia, leve ameaça do desequilíbrio, imediatamente o SNC recruta, via entrada somatossensorial interna e externa, os músculos dos tornozelos para correção e/ ou manutenção do controle postural. ${ }^{19}$ Diante disso, há ligeira liberação (relaxamento) da musculatura do tronco e consequente enrijecimento da articulação do tornozelo. ${ }^{23}$ Essa tática postural pode ser considerada um reflexo do subconsciente. Vale ressaltar que, por causa do aumento da idade, idosos apresentam diminuição gradual da força dos músculos estabilizadores do tornozelo, o que obriga o organismo executar, automaticamente, a próxima estratégia;

- Fase $1 \mathrm{~b}$ (estratégia do quadril): caso forças maiores atuem sobre o corpo, ameaçando a estabilidade da postura ereta, o SNC solicita a estratégia do quadril. ${ }^{19}$ Esse mecanismo incide na ativação tardia dos músculos do tronco e da coxa, irradiando forças em sentido proximal-distal. ${ }^{13}$ Comparativamente, pode-se dizer que a estratégia do tornozelo exige a ativação dos flexores plantares do tornozelo, flexores do joelho e extensores do quadril, enquanto a estratégia do quadril requer a ativação dos extensores do joelho e flexores do quadril. Conforme Blenkinsop et al., ${ }^{23}$ a estratégia do tornozelo é geralmente aplicada em situações da postura não perturbada rumo a perturbações de baixa amplitude. Por outro lado, a estratégia do quadril é solicitada em circunstâncias de perturbação rápida com grande amplitude. Um exemplo disso é quando o indivíduo se encontra sobre uma superfície 
estreita de apoio, que dificulta a aplicação do torque do tornozelo;

- Fase 2 (estratégia do passo): quando ocorrem episódios de grande intensidade e mecanismos anteriores não foram capazes de reestabelecer o balanço e o desequilíbrio é detectado, o SNC interrompe automaticamente as estratégias do tornozelo e do quadril e recruta a estratégia do passo. ${ }^{19} \mathrm{O}$ procedimento é efetuado pelo envio de mensagens às extremidades, paralelamente, o SNC solicita que a base de suporte seja reposicionada, para tanto é executado um passo para frente, traz ou lado. ${ }^{13}$ Durante o processo, a articulação do tornozelo é liberada, permitindo a ampliação da base de suporte do corpo;

- Fase 3 (próximo à queda): se por acaso, ações motoras anteriores ainda não tenham conseguido preservar a estabilidade postural, o SNC dispara os membros superiores como tática de resgate da postura. A ação é reflexiva e busca, acima de tudo, evitar e/ou minimizar o acidente eminente. ${ }^{13,19}$ Do ponto de vista da

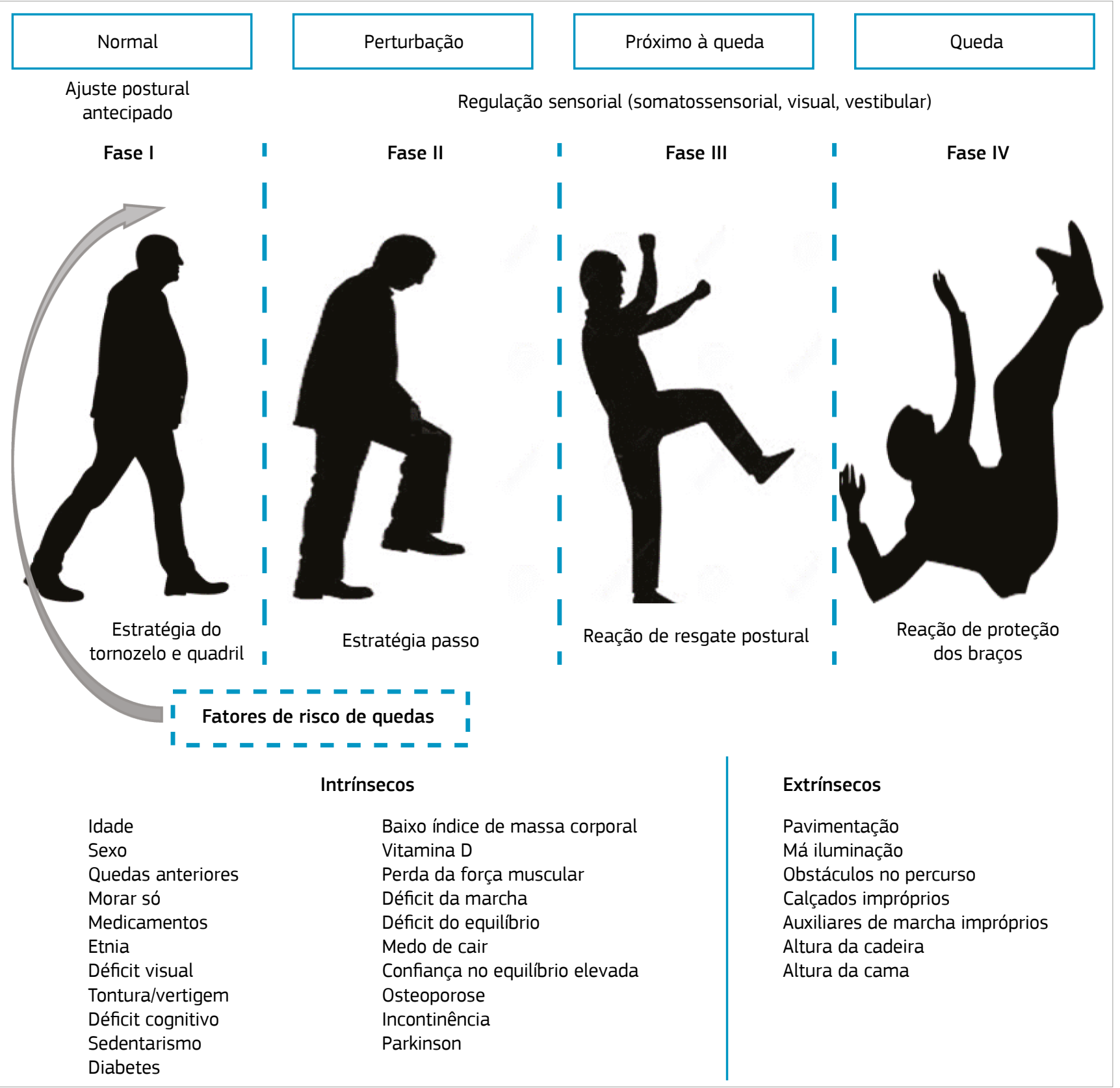

Figura 1 Modelo sobre a representação da sequência de estratégias aplicadas à preservação da estabilidade do corpo após uma perturbação. Esquema criado com base nos estudos de Montero-Odasso e Speechley ${ }^{13}$ e Fasano et al. ${ }^{19}$ 
cinemática, em situações próximas à queda, independentemente da estratégia empregada pelo SNC, tanto a movimentação do corpo como o torque aplicado sobre o tornozelo e/ou quadril são inevitáveis. ${ }^{23}$ Nesse contexto, a aceleração de um segmento determinará a aceleração dos demais segmentos, que devem ser controlados pela musculatura apropriada. ${ }^{24}$ Enquanto isso, o $\mathrm{SNC}$ emite estímulos determinando torques compensatórios do quadril na direção do tornozelo, aumentando a velocidade da movimentação das pernas. A estratégia do quadril também gera torque complementar sobre o tornozelo, na direção oposta ao torque do quadril, produzindo a rotação dos membros superiores e inferiores. ${ }^{23}$

De forma geral, a sequência das estratégias apresentadas na Figura 1 surge em resposta ao fluxo inadequado de informações posturais de aferência visual, vestibular e somatossensorial. ${ }^{9,13}$ Isso significa dizer que o encadeamento das ações empregadas consiste em uma resposta às disfunções sensoriais e executivas apresentadas pelo organismo para se adaptar às circunstâncias que conduzem o indivíduo à queda. $\mathrm{O}$ fato pode ser detalhado como a incapacidade do organismo (sistema sensorial do equilíbrio corporal) para reagir, a tempo, contra forças de perturbação externa. ${ }^{14,15}$

A literatura especializada indica que além dos déficits neurais responsáveis pela regulação do equilíbrio, o medo de cair também potencializa significativamente o risco de queda de adultos idosos. Estima-se que o receio de cair aumente em $25 \%$ o risco de queda, principalmente entre caidores recorrentes. ${ }^{16,25}$ Young e Williams ${ }^{26}$ destacaram que o medo de queda gera ansiedade, diminuindo os procedimentos de atenção necessários à manutenção do equilíbrio, afetando também a velocidade da marcha. Tudo isso compromete consideravelmente o planejamento seguro das tarefas locomotoras de ordem complexa.

Fasano et al. ${ }^{19}$ destacaram que, embora a marcha e a estabilidade postural sejam procedimentos automáticos (subcorticais), o conjunto de estratégias utilizadas para gerenciar as perturbações posturais está intimamente associado às funções corticais. Assim, a manutenção da posição ereta, além de carecer do fluxo adequado de informações obtidas por inputs sensoriais de aferência somatossensorial, visual e vestibular, também requer recursos cognitivos.

Estudo de revisão sistemática desenvolvido por Kearney et al. ${ }^{27}$ constatou, em idosos da comunidade, associação entre quedas com a baixa da velocidade da marcha e déficit do desempenho das funções executivas (Figura 1). Conforme os autores, as funções executivas são responsáveis pelo controle inibitório, atenção, memória operacional e flexibilidade cognitiva. As funções executivas desempenham forte papel no processamento das informações posturais, de tal modo, déficits cognitivos geram lentidão à mobilidade humana..$^{23-25}$

Vale ressaltar que até mesmo idosos classificados com desempenho cognitivo normal podem apresentar problemas de disfunção executiva. Essa linha de raciocínio evidencia a importância da identificação precoce de idosos "caidores idiopáticos”. Por essa razão, a prática clínica utiliza um conjunto de instrumentos específicos para o exame de déficits das funções executivas: ${ }^{28}$ Verbal Fluency, Trail Making Test (TMT) Montreal Cognitive Assessment (MoCA), Clock Drawing Test (CDT) ou o Miniexame do Estado Mental (MEEM).

\section{CONTRIBUIÇÕES DO EXERCÍCIO FÍSICO Ả PRẺVENÇÃO DE QUEDAS}

Conforme o American College of Sports Medicine, ${ }^{29}$ o exercício é um movimento corporal executado de forma planejada, estruturada e repetitiva com o objetivo de melhorar ou manter um ou mais componentes da aptidão física. Estudos de revisão sistemática ${ }^{30}$ e metanálise ${ }^{31}$ mostraram que o exercício físico, quando realizado de forma regular e planejada, é capaz de reduzir significativamente as taxas de queda da população idosa. Seus efeitos mantêm e/ou melhoram o desempenho do sistema fisiológico, refletindo positivamente sobre a funcionalidade e mobilidade do indivíduo, qualificando a resolução das atividades da vida diária. Aliado ao fato, o exercício físico é bem aceito pela população idosa. ${ }^{32}$

Estudo atual de revisão sistemática ${ }^{33}$ mostrou que exercícios realizados por meio da dança, exergaming e modalidades esportivas com bolas podem retardar o declínio cognitivo. Isso ocorre porque essas atividades beneficiam áreas do cérebro responsáveis por domínios múltiplos, contribuindo para o resgate do equilíbrio durante a marcha (Figura 1). Em outro estudo atual de revisão sistemática e metanálise composto por 88 ensaios (19.478 indivíduos), Sherrington et al. ${ }^{17}$ mostraram que a prática do exercício físico foi capaz de reduzir em $21 \%$ a taxa de queda de adultos idosos. Os autores observaram que o maior efeito sobre quedas ocorreu em grupos que praticavam exercícios que desafiavam o equilíbrio com duração $\geq 3$ horas semanais. Ademais, verificou-se que a prática regular do exercício físico também apresentou efeito preventivo sobre quedas entre idosos parkinsonianos ou com comprometimento cognitivo.

Tratando-se do melhor tipo de exercício a ser adotado para a prevenção de queda de adultos idosos, a literatura especializada não apresenta um consenso. ${ }^{25} \mathrm{Sabe-se}$, porém, que 
o exercício físico apresenta vantagens quando executado de forma estruturada, conforme programas. ${ }^{34,35}$ Os benefícios sobrevêm do planejamento, da frequência e da progressão exata das atividades. Entre os programas, a literatura internacional cita o Otago ${ }^{36}$ como procedimento capaz de reduzir a taxa de queda em $35 \%$. Esse programa apresenta vantagens, em especial para indivíduos com limitações de deslocamento até o local de realização das atividades em grupo, visto que as atividades podem ser desenvolvidas no próprio domicílio do idoso. O programa também prevê cinco visitas por profissionais da área da fisioterapia ou enfermagem à casa dos idosos. Esses são encarregados de ensinar e supervisionar as atividades. Além do mais, as ações são seguidas por um acompanhamento telefônico mensal.

\section{EQUILÍBRIO}

Lahr et al. ${ }^{21}$ verificaram níveis de oscilações na base de suporte tanto de indivíduos idosos $(75,4 \pm 5,7$ anos) como de adultos jovens $(22,6 \pm 2,33$ anos). Os autores constataram, todavia, maior oscilação entre os idosos. Ademais, os idosos mostraram restrições no funcionamento dos três sistemas de regulação sensorial do equilíbrio, o que limitou o desempenho de tarefas durante a progressão das dificuldades (permanecer sobre a plataforma de força 30 segundos nas bases de suporte normal e semitandem).

Estudo de metanálise desenvolvido por Sherrington et al. ${ }^{37}$ relatou que programas de exercícios planejados foram capazes de reduzir a taxa de queda em até $42 \%$. De acordo com os autores, destacaram-se as atividades para se equilibrar com nível de desafio de moderado a alto, praticadas com frequência mínima de 2 horas semanais, durante 6 meses. Os autores também aconselharam a associação do treinamento de força e de caminhada com medidas educativas: esse procedimento é essencial à transformação dos hábitos de vida.

Com base nos 57 estudos analisados por Sherrington et al. ${ }^{37}$ os seguintes procedimentos foram recomendados para o treinamento de prevenção de quedas:

- redução da base de suporte: ficar de pé com as pernas juntas, com um pé à frente do outro (tandem) e se possível sobre uma perna;

- movimentos com perturbação do centro de gravidade: caminhar em tandem, transferir o peso corporal de uma perna para a outra, ultrapassar obstáculos, assim como subir em objetos estáveis;

- treinamento de resistência específica para grupos musculares posturais: agachamento, abdução do quadril (com pesos adicionais);
- redução da entrada sensorial: praticar exercícios com os olhos fechados, caminhar para frente e de costas sobre superfícies instáveis e/ou reduzidas;

- incremento de desafios por intermédio de atividades de dupla tarefa: associar tarefas de memória, atenção e cálculo com tarefas de marcha, coordenação e equilíbrio estático e dinâmico.

Ghai et al. ${ }^{22}$ avaliaram 1.284 estudos, desses, 42 investigações (1.480 participantes) foram selecionadas. Os autores destacaram os efeitos do treinamento de dupla tarefa, salientando que verificaram, em 87,5\% dos trabalhos, $30 \%$ de efeito agudo do treinamento de dupla tarefa sobre a estabilidade postural. Os achados foram ratificados por uma metanálise que mostrou efeitos moderados (intervalo de confiança de 95\% 1,16-2,10) e significativos para redução dos riscos de queda de idosos da comunidade.

\section{FORÇA}

Um dos efeitos negativos do processo do envelhecimento sobre o organismo humano é a sarcopenia. Essa alteração fisiológica é gerada pelo estresse oxidativo, que baixa a produção de testosterona no organismo. ${ }^{38}$ Diante disso, decresce a produção natural de substâncias anabólicas e, por conseguinte, seus efeitos sobre o tecido músculo esquelético. Por essa razão, há aceleração do processo de atrofia dos tecidos e redução dos níveis de força muscular. Tudo isso potencializa a perda da massa muscular. No entanto, por meio do treinamento de força é possível que o organismo de adultos idosos consiga amenizar esse processo fisiológico.

Por meio da sobrecarga do exercício sobre os músculos, há aumento da velocidade de contração muscular. Por conseguinte, o indivíduo é capaz de reagir rapidamente em situações de desequilíbrio (Figura 1). O controle postural consiste em um sistema integrado, melhorando a velocidade de contração muscular, o SNC é capaz de agilizar as informações corretivas, emitindo de forma mais segura informações às extremidades do corpo. Estudos anteriores ${ }^{18,39}$ realizados com adultos idosos mostraram que 12 semanas de treinamento resistido, composto por 3 a 4 séries, com 12 a 15 repetições e ajuste da carga sempre que relatada como leve proporcionaram aumento significativo dos níveis de força. Paralelo ao fato, observou-se melhora significativa do desempenho em testes de marcha e equilíbrio funcional.

Por outro lado, Orsano et al. ${ }^{40}$ destacaram que, em se tratando da população idosa, o treinamento de potência é mais eficaz do que o treino de força tradicional (lento ou moderado). Pois no treinamento de potência, a fase concêntrica é 
realizada de forma mais veloz, o que proporciona a ativação de um número maior de unidades motoras e, por conseguinte, melhor adaptação das fibras do tipo II. Conforme os autores, essa técnica pode ser praticada em máquinas ou com o auxílio de pesos livres e elásticos; recomenda-se a execução da fase concêntrica em tempo $<1$ segundo e a fase excêntrica, em aproximadamente 2 ou 3 segundos.

\section{MOBILIDADE}

Com o avanço da idade também existem alterações junto ao padrão da marcha. Estudos de revisão sistemática relataram baixa da velocidade e aumento da variabilidade da passada (tempo da passada, largura do passo e tempo de suporte duplo) em adultos idosos, principalmente em situações que exigiram a atenção dividida. ${ }^{41,42}$ Por outro lado, sabe-se que o treinamento de dupla tarefa é capaz de reverter o quadro, aumentando tanto a velocidade da marcha como o comprimento e a variabilidade do tempo da passada..$^{22,43}$

Ciprandi et al. ${ }^{44}$ verificaram, em estudo transversal desenvolvido com mulheres saudáveis (65 a 75 anos), associação significativa e negativa entre a variabilidade da marcha e o nível de atividade física. Isso significa dizer que o aumento dos níveis de aptidão física do idoso podem minimizar o risco de queda. Em estudo observacional realizado com 103 indivíduos (76,3 $\pm 7,2$ anos), sendo $56 \%$ mulheres portadoras de distúrbios da marcha e déficit de memória, Auvinet et al. ${ }^{45}$ constataram instabilidade postural mediante a análise da marcha na condição de dupla tarefa. Os autores também associaram o desequilíbrio postural com quatro subgrupos patológicos: doenças musculoesqueléticas; vestibulopatias; comprometimentos cognitivos leves; e patologias do SNC.

\section{CONCLUSÃO}

Com base nas informações exibidas pelo presente estudo, conclui-se que profissionais da área clínica comprometidos com a prevenção de queda de adultos idosos devem aprofundar seus conhecimentos sobre a intersecção entre o risco de queda e fatores associados, bem como sobre a fisiopatologia responsável por quedas. ${ }^{10,13}$ Conforme as informações detalhadas, sugere-se especial atenção a indivíduos portadores de comprometimentos cognitivos. Quanto à prática regular de exercícios físicos, observou-se a necessidade de que esses privilegiem atividades devidamente planejadas e estruturadas, ${ }^{30,35}$ incluindo métodos que agreguem tarefas cognitivas. ${ }^{22,28}$

\section{CONFLITO DE INTERESSES}

Os autores declaram não haver conflito de interesses.

\section{REFERÊNCIAS}

1. Talarska D, Strugała M, Szewczyczak M, Tobis S, Michalak M, Wróblewska I, et al. Is independence of older adults safe considering the risk of falls? BMC Geriatr. 2017;17(1):66. http://doi.org/10.1186/s12877017-0461-0

2. Sherrington C, Tiedemann A, Fairhall NJ, Hopewell S, Michaleff ZA, Howard $\mathrm{K}$, et al. Exercise for preventing falls in older people living in the community. Cochrane Database Syst Rev. 2016;(11):1-15. http:// doi.org/10.1002/14651858.CD012424

3. Chang VC, Do MT. Risk factors for falls among seniors: Implications of gender. Am J Epidemiol. 2015;181(7):521-31. http://doi.org/10.1093/ aje/kwu268

4. Organizaçao Mundial da Saúde. Relatório Mundial de Envelhecimento e Saúde. Genebra: Organizaçao Mundial da Saúde; 2015.

5. Verma SK, Willetts JL, Corns HL, Marucci-Wellman HR, Lombardi DA, Courtney TK. Falls and fall-related injuries among community-dwelling adults in the United States. PLoS One. 2016;11(3):e0150939. http:// doi.org/10.1371/journal.pone.0150939

6. Barros IFO, Pereira MB, Weiller TH, Anversa ETR. Internações hospitalares por quedas em idosos brasileiros e os custos correspondentes no âmbito do Sistema Único de Saúde. Rev Kairós Gerontol. 2015;18(4):63-80.

7. Lusardi MM, Fritz S, Middleton A, Allisson L, Wingood M, Phillips $E$, et al. Determining Risk of falls in community dwelling older adults: A systematic review and meta-analysis using posttest probability. J Geriatr Phys Ther. 2017;40(1):1-36. http://doi.org/10.1519/ JPT.0000000000000099

8. Tinetti M. Preventing Falls in Elderly Persons. N Engl J Med. 2003;348:42-9. http://doi.org/10.1056/NEJMcp020719

9. Januário F, Amaral IC. Fisiologia do equilíbrio. Rev Soc Port Med Física Reabil. 2010;19(2):31-7.
10. Terroso M, Rosa N, Torres Marques A, Simões R. Physical consequences of falls in the elderly: A literature review from 1995 to 2010. Eur Rev Aging Phys Act. 2014;11(1):51-9. http://doi.org/10.1007/s11556-013-0134-8

11. Toledo DR, Barela JA. Sensory and motor differences between young and older adults: somatosensory contribution to postural control. Rev Bras Fisioter. 2010;14(3):267-75.

12. Błaszczyk J, Michalski A. Ageing and postural stability. Stud Phys Cult Tour. 2006;13(Sup.):11-4.

13. Montero-Odasso M, Speechley M. Falls in Cognitively Impaired Older Adults: Implications for Risk Assessment And Prevention. J Am Geriatr Soc. 2018;66(2):367-75. http://doi.org/10.1111/jgs.15219

14. Yuki K, Asaoka R, Tsubota K. Investigating the influence of visual function and systemic risk factors on falls and injurious falls in glaucoma using the structural equation modeling. PLoS One. 2015;10(6):e0129316. http://doi.org/10.1371/journal.pone.0129316

15. Kleiner AFR, Schlitter DXDC, Sanchés-Arias MDR. O papel dos sistemas visual, vestibular, somatosensorial e auditivo para o controle postural. Rev Neurociências. 2011;19(2):349-57.

16. Lavedán A, Viladrosa M, Jürschik P, Bótigue T, Nuín C, Masot O, et al. Fear of falling in community-dwelling older adults: A cause of falls, a consequence, or both? PLoS One. 2018;13(3):e0194967. http://doi. org/10.1371/journal.pone.0194967

17. Sherrington C, Michaleff ZA, Fairhall N, Paul SS, Tiedemann A, Whitney $\mathrm{J}$, et al. Exercise to prevent falls in older adults: An updated systematic review and meta-analysis. Br J Sports Med. 2017;51(24):1750-8. http://doi.org/10.1136/bjsports-2016-096547

18. Tricco AC, Thomas SM, Veroniki AA, Hamid JS, Cogo E, Strifler S, et al. Comparisons of interventions for preventing falls in older adults: A systematic review and meta-analysis. J Am Med Assoc 2017;318(17):1687-99. http://doi.org/10.1001/jama.2017.15006 
19. Fasano A, Plotnik M, Bove F, Berardelli A. The neurobiology of falls. Neurol Sci. 2012;33(6):1215-23. http://doi.org/10.1007/s10072012-1126-6

20. Panel on Prevention of Falls in Older Persons, American Geriatrics Society and British Geriatrics Society. Summary of the updated american geriatrics society/British geriatrics society clinical practice guideline for prevention of falls in older persons. J Am Geriatr Soc. 2011;59(1):148-57. http://doi.org/10.1111/j.1532-5415.2010.03234.x

21. Lahr SLN, Ungrinowitsch H, Dos Santos LLP, De Andrade AGP, Benda RN. Efeitos do envelhecimento e da base de suporte no controle postural. Rev Bras Educ Fís Esporte. 2017;31(1):83-90. https://doi. org/10.11606/1807-5509201700010083

22. Ghai S, Ghai I, Effenberg AO. Effects of dual tasks and dual-task training on postural stability: A systematic review and meta-analysis. Clin Interv Aging. 2017;12(1):557-77. http://doi.org/10.2147/CIA.S125201

23. Blenkinsop GM, Pain MTG, Hiley MJ. Balance control strategies during perturbed and unperturbed balance in standing and handstand. R Soc Open Sci. 2017;4(7). http://doi.org/10.1098/rsos.161018

24. Horak FB, Diener HC, Nashner LM. Influence of central set on human postural responses. J Neurophysiol. 1989;62(4):841-53. http://doi. org/10.1152/jn.1989.62.4.841

25. Svantesson U, Babagbemi B, Foster L, Alricsson M. Influences on modern multifactorial falls prevention Interventions and fear of falling in non-frail older adults: A literature review. J Clin Med Res. 2014;6(5):314-20. http://doi.org/10.14740/jocmr1874w

26. Young WR, Williams AM. How fear of falling can increase fall-risk in older adults: Applying psychological theory to practical observations. Gait Posture. 2015;41(1):7-12. http://doi.org/10.1016/j.gaitpost.2014.09.006

27. Kearney FC, Harwood RH, Gladman JRF, Lincoln N, Masud T. The relationship between executive function and falls and gait abnormalities in older adults: A systematic review. Dement Geriatr Cogn Disord. 2013;36(1-2):20-35. http://doi.org/10.1159/000350031

28. Lima LCA, Ansai JH, Andrade LP, Takahashi ACM. The relationship between dual-task and cognitive performance among elderly participants who exercise regularly. Brazilian J Phys Ther. 2015;19(2):159-66. http:// doi.org/10.1590/bjpt-rbf.2014.0082

29. American College of Sports Medicine. American College of Sports Medicine Position Stand. Progression models in resistance training for healthy adults. Med Sci Sports Exerc. 2009;41(3):687-708. https:/l doi.org/10.1249/MSS.0b013e3181915670

30. Shier V, Trieu E, Ganz DA. Implementing exercise programs to prevent falls: systematic descriptive review. Inj Epidemiol. 2016;3:16. http:// doi.org/10.1186/s40621-016-0081-8

31. Plummer P, Zukowski LA, Giuliani C, Hall AM, Zurakowski D. Effects of Physical Exercise Interventions on Gait-Related Dual-Task Interference in Older Adults: A Systematic Review and Meta-Analysis. Gerontology. 2015;62(1):94-117. http://doi.org/10.1159/000371577

32. Davis JC, Bryan S, Best JR, Li LC, Hsu CL, Gomez C, et al. Mobility predicts change in older adults' health-related quality of life: evidence from a Vancouver falls prevention prospective cohort study. Health Qual Life Outcomes. 2015;13:101. http://doi.org/10.1186/s12955-015-0299-0
33. Muiños M, Ballesteros S. Does physical exercise improve perceptual skills and visuospatial attention in older adults? A review. Eur Rev Aging Phys Act. 2018;15:2. http://doi.org/10.1186/s11556-018-0191-0

34. Tiedemann A, Sherrington C, Lord SR. The role of exercise for fall prevention in older age. Motriz Rio Claro. 2013;19(3):541-7. http:// doi.org/10.1590/S1980-65742013000300002

35. Nascimento MM. Problematizando quedas em idosos: Estrutura conceitual à organização de programas de exercícios físicos. Rev Interdiscip Promoçã̃o Saúde. 2018;1(2):83-91. http://dx.doi. org/10.17058/rips.v1i2.12316

36. Campbell AJ, Norton R, Robertson C. Randomized controlled trial of a general practice programme of home based exercise to prevent falls in elderly women. J Am Geriatr Soc. 1997;315(7115):1065-9. https://doi.org/10.1136/bmj.315.7115.1065

37. Sherrington C, Tiedmann NF, Fairhall N, Close JCT, Lord SR. Exercise to prevent falls in older adults: an updated meta-analysis and best practice recommendations. N S W Public Health Bull. 2011;22(34):78-83. https://doi.org/10.1071/NB10056

38. Leite LEDA, Resende TDL, Nogueira GM, Cruz IBM, Schneider RH, Gottlieb MGV. Envelhecimento, estresse oxidativo e sarcopenia: uma abordagem sistêmica. Rev Bras Geriatr e Gerontol. 2012;15(2):36580. http://doi.org/10.1590/S1809-98232012000200018

39. Souza LK, Coelho BS, Freire B, Delevatti RS, Roncada C, Tiggemann CL, et al. Comparação dos níveis de força e equilíbrio entre idosos praticantes de musculação e de hidroginástica. Rev Bras Atividade Física Saúde. 2014;19(5):647-55. https://doi.org/10.12820/rbafs.v.19n5p647

40. Orsano VSM, Moraes WMAM, Prestes J. Treinamento de potência em idosos: porque é importante? Rev Bras Ciên Mov. 2017;25(4):181-7. http://dx.doi.org/10.18511/rbcm.v25i4.8661

41. Gomes GC, Teixeira-Salmela LF, Freitas FAS, Fonseca MLM, Pinhero $\mathrm{MB}$, Morais VAC, et al. Gait performance of the elderly under dualtask conditions: Review of instruments employed and kinematic parameters. Rev Bras Geriatr Gerontol. 2016;19(1):165-82. http:// doi.org/10.1590/1809-9823.2016.14159

42. Fraser SA, Li KZ-H, Berryman N, Dejardins-Crépau L, Lussier M, Vadaga K, et al. Does Combined Physical and Cognitive Training Improve Dual-Task Balance and Gait Outcomes in Sedentary Olde Adults? Front Hum Neurosci. 2017;10:1-12. http://doi.org/10.3389/ fnhum.2016.00688

43. Dorfman M, Herman T, Brozgol M, Shema S, Weiss A, Hausdorff JM, et al. Dual-task training on a treadmill to improve gait and cognitive function in elderly idiopathic fallers. J Neurol Phys Ther. 2014;38(4):24653. http://doi.org/10.1097/NPT.0000000000000057

44. Ciprandi D, Bertozzi F, Zago M, Ferreira CLP, Boari G, Sforza C, et al. Study of the association between gait variability and physical activity. Eur Rev Aging Phys Act. 2017;14:19. http://doi.org/10.1186/s11556017-0188-0

45. Auvinet B, Touzard C, Montestruc F, Delafond A, Goeb V. Gait disorders in the elderly and dual task gait analysis: a new approach for identifying motor phenotypes. J Neuroeng Rehabil. 2017;14:7. http:// doi.org/10.1186/s12984-017-0218-1 